\title{
SARS-COV-2 VIROPORINS ACTIVATE THE NLRP3-INFLAMMASOME VIA THE MITOCHONDRIAL PERMEABILITY TRANSITION PORE
}

Joseph W. Guarnieri ${ }^{1}$, Alessia Angelin ${ }^{1}$, Deborah G. Murdock ${ }^{1}$, Prasanth Portluri ${ }^{1}$, Timothy Lie ${ }^{1,3}$, Douglas C. Wallace $^{1,2 *}$.

${ }^{1}$ Center for Mitochondrial and Epigenomic Medicine, Division of Human Genetics, The Children's Hospital of Philadelphia, Philadelphia, PA 19104, USA.

${ }^{2}$ Department of Pediatrics, Division of Human Genetics, Perelman School of Medicine, University of Pennsylvania, Philadelphia, PA 19104, USA.

${ }^{3}$ University of Pennsylvania, Philadelphia, PA 19104 USA

${ }^{*}$ Correspondence: WallaceD1@chop.edu

\section{SUMMARY}

Cytokine storm precipitated by activation of the host innate immune defenses is a major cause of COVID19 death. To elucidate how SARS-CoV-2 initiates this inflammatory process, we studied viroporin proteins $E$ and Orf3a (2-E+2-3a). Expression of 2-E+2-3a in human 293T cells resulted in increased cytosolic $\mathrm{Ca}^{++}$and then elevated mitochondrial $\mathrm{Ca}^{++}$, taken up through the MUCi11-sensitive mitochondrial calcium uniporter (MCU). Increased mitochondrial $\mathrm{Ca}^{++}$resulted in stimulation of mitochondrial reactive oxygen species (mROS) production, which was blocked by mitochondrially-targeted catalase or MnTBAP. To determined how mROS activates the inflammasome, we transformed 293T cells with NLRP3, ASC, pro-caspase-1 and pro-IL-1 $\beta$ plus used THP1 derived macrophages to monitor the secretion of mature IL-1 3 . This revealed that mROS activates a factor that is released via the NIM811-sensitive mitochondrial permeability pore (mtPTP) to activate the inflammasome. Hence, interventions targeting mROS and the mtPTP may mitigate the severity of COVID19 cytokine storms.

Keywords: SARS-CoV-2, COVID-19; cytokine storm; NLRP3-inflammasome; ORF3a; envelope (E); viroporin; mitochondrial permeability transition pore; mitochondrial reactive oxygen species (mROS); mitochondria in innate immune responses. 


\section{INTRODUCTION}

Approximately 350 million cases of COVID19 have been reported globally, resulting in over 5.5 million deaths (Dong et al., 2020). COVID19 is caused by SARS-CoV-2 whose genome structure encodes a polyprotein that is cleaved into 16 non-structural proteins (nsp) as well as the structural proteins $S$ (Spike), E (Envelope), M (Membrane), and $\mathrm{N}$ (Nucleocapsid), and seven open reading frames (orfs) 3a, 6, 7a, 7b, 8, 9b, and 10, with substantial homology with SARS-CoV-1.

Severe COVID19 manifests as pneumonia, acute respiratory distress syndrome, respiratory failure, and cytokine storm resulting in multiple organ failure (Ferreira et al., 2021; Rodrigues et al., 2021; Yang et al., 2021). The cytokine storm results from the elaboration of pro-inflammatory cytokines such as interleukin (IL)-1 1 (Ajaz et al., 2021; Chen et al., 2020; Chi et al., 2020; Han et al., 2020; Lucas et al., 2020; Wen et al., 2020).

The production of mature IL-1 $\beta$ requires the activation of the mitochondrially-bound NLRP3inflammasome (NLRP3-I), which encompasses the NLR family pyrin domain containing 3 (NLRP3) receptor; the adaptor molecule apoptosis-associated speck-like protein containing a caspase activation and recruitment domain (ASC); and the pro-IL-1 $\beta$-converting enzyme pro-caspase-1 (CASP1). Upon activation, the NLRP3-I triggers the proteolytic cleavage of pro-caspase-I (pro-CASP1), and CASP1 cleaves pro-IL-1 $\beta$ to generate IL-1 $\beta$ which is secreted from the cell (Broz and Dixit, 2016). Autopsy samples from severe COVID19 patients display increased NLRP3-I activation in lung tissues and peripheral blood mononuclear cells (Rodrigues et al., 2021), and monocytes isolated from severe COVID19 patients have increased levels of activated NLRP3-I and IL-1 $\beta$, (Ferreira et al., 2021). Thus, understanding the mechanism by which SARS-CoV-2 activates the NLRP3-I is imperative for understanding the pathophysiology of severe COVID19.

Recently, mitochondrial dysfunction has been shown to activate the innate immune system via mitochondrial reactive oxygen species (ROS) production and oxidation of the mitochondrial DNA (mtDNA) during replication, induced by the expression of the rate-limiting enzyme cytosine monophosphate kinase 2 (CMPK2). The oxidized mtDNA (Ox-mtDNA) is released from the mitochondrion to bind and activate the NLRP3-I (West and Shadel, 2017; Zhong et al., 2018). While the mechanism by which SARS-CoV-2 activates the NLRP3-I is unknown, expression of the SARS-CoV-1/2 viroporins have been associated with activation of NLRP3-I (Chen et al., 2019, Nieto-Torres, 2015 \#118; Siu et al., 2019; Xia et al., 2021; Yue et al., 2018) and are known to be membrane ion channels (Hover et al., 2017; Nieva et al., 2012).

SARS-CoV-2 encodes two viroporins E (2-E) (Verdiá-Báguena et al., 2021) and ORF3a (2-3a) (Qu et al., 2021), with homologues to the SARS-CoV-1 proteins (Kern et al., 2021; Mandala et al., 2020). SARS-CoV-1/2 $E$ and 3 a viroporins localize to the endoplasmic reticulum (ER), Golgi apparatus, and plasma membrane (Gordon et al., 2020a) where they increase the permeability to cations such as $\mathrm{Ca}^{++}$(Minakshi and Padhan, 2014; VerdiáBáguena et al., 2021, Kern, 2021 \#420; Verdiá-Báguena et al., 2012). For SARS-CoV-1, the 1-E and 1-3a have been shown to activate the NLRP3-I in human monocyte-derived macrophages (Chen et al., 2019; Siu et al., 2019; Yue et al., 2018). In LPS-primed macrophages, co-expression of 1-E plus 1-3a resulted in higher levels of IL-1 $\beta$ secretion than either viroporin alone (Chen et al., 2019), 1-E has been reported to activate NLRP3-I through disrupting $\mathrm{Ca}^{++}$homeostasis in cells (Nieto-Torres et al., 2015; Xia et al., 2021), and activation of the NLRP3-I and secretion of IL-1 $\beta$ by $1-E$ and $1-3 a$ is mitigated by treatment with the mROS scavenger MitoQ (Chen et al., 2019). However, the mechanism by which SARS-CoV-2 activates the NLRP3-I has yet to be explained.

We hypothesized that expression of 2-E plus 2-3a results in increased $\mathrm{Ca}^{++}$flux into the cytosol where it is taken up by the mitochondrion through the mitochondrial $\mathrm{Ca}^{++}$uniporter (MCU). Within the mitochondrion, the $\mathrm{Ca}^{++}$activates the pyruvate and $\alpha$-ketoglutarate dehydrogenases to generate excessive NADH (Denton, 2009). The increased NADH overloads the mitochondrial electron transport chain producing increased mitochondrial ROS (mROS). The mROS oxidizes the mtDNA, and the Ox-mtDNA is released through the mitochondrial permeability transition pore (mtPTP) to bind to the NLRP3 inflammasome. This activates caspase-1 to cleave pro-IL-1 $\beta$ resulting in the secretion of active IL-1 $\beta$ (Xian et al., 2021; Zhong et al., 2018). Our current results support this scenario, thus placing mitochondrial function at the nexus between SARS-CoV-2 infection and the cytokine storm of severe COVID19. 


\section{RESULTS}

\section{Expression of 2-E+2-3a increases $\mathrm{Ca}^{++}$leakage into the cytosol, elevates mitochondrial Ca ${ }^{++}$levels, and increases $\mathrm{mROS}$ production.}

We constructed a polycistronic expression vector combining 2-E+2-3a (LV-E3a) (Figure 1A\&B). In this vector the 2-E+2-3a sequences were separated by the self-cleaving $2 A$ peptide site (Liu et al., 2017; SzymczakWorkman et al., 2012) to allow co-expression from a single transcript. The expression of 2-E+2-3a in LV-E3a transduced 293T cells was confirmed by Western blot (Figure 1C). We next demonstrated that LV-E3a transduced 293T cells experience increased cytosolic $\mathrm{Ca}^{++}$with Fura-Red (Figure 1D-F) and mitochondrial Ca ${ }^{++}$ with Rhod2 (Figure 1G-I). Thapsigargin (TG), which triggers $\mathrm{Ca}^{++}$flux into the cytosol, increased mitochondrial $\mathrm{Ca}^{++}$uptake (Bagur and Hajnóczky, 2017; Csordás et al., 2018), and mitochondrial Ca ${ }^{++}$uptake was increased in TG treated LV-E3a transduced cells. Co-treatment with the mitochondrial calcium uniporter inhibitor 11 (MCUi11) (Di Marco et al., 2020; Márta et al., 2021) abolished mitochondrial calcium uptake (Figure 1J). Thus, expression of 2-E+2-3a in 293T cells results in elevated cytosolic $\mathrm{Ca}^{++}$which is taken up by the mitochondrial calcium uniporter resulting in elevated mitochondrial $\mathrm{Ca}^{++}$(Figure 1J).

We next demonstrated that 2-E+2-3a expression in 293T cells increased mROS production by staining transduced cells with MitoSOX, which detects mitochondrial superoxide anion production. MitoSOX fluorescence confirmed that 2-E+2-3a expression increased mROS production (Figure 1K-M). To determine if the increased mROS was due to the entry of $\mathrm{Ca}^{++}$into the mitochondrion, we treated the cells with MCUi11 which blocked the increased $\mathrm{mROS}$ production (Figure 1N).

To confirm that the $2-E+2-3 a$ induced ROS production was mROS, we transformed the 2-E+2-3a expressing 293T cells with a vector expressing mitochondrially-targeted catalase (mCAT) (Figure 2A-C) which removes mitochondrial $\mathrm{H}_{2} \mathrm{O}_{2}$ (Schriner et al., 2005), or treated the cells with the mitochondrially targeted catalytic metalloporphyrin anti-oxidant, MnTBAP (Melov et al., 1998; Tong et al., 2007). Treatment with either MnTBAP or mCAT extinguished the $2-E+2-3 a$ activated ROS product, confirming that the ROS was generated by the mitochondrion (Figure 2D-I).

\section{2-E+2-3a induced $m R O S$ is involved in NLRP3-activated and IL-1 $\beta$ production.}

In SARS-CoV-1, activation of the NLRP3-I and pathogenicity are associated with both 1-E+1-3a (Chen et al., 2019; Nieto-Torres et al., 2014; Xia et al., 2021; Zhang et al., 2021). To determine if this is the case for SARS-CoV-2, we used two model systems to determine if 2-E+2-3a expression activates the NLRP3-I via the mitochondrion. First, we transformed 293T cells with plasmids encoding the components of the NLRP3-I, thus reconstituting the inflammasome (Shi et al., 2016) (Figure 3A). Second, we transduced THP1 cells which are a human acute monocytic leukemia derived cell line with the 2-E+2-3a expression vector. The transduced THP1 cells were then treated with phorbol ester (PMA) to generate macrophages and the macrophages were treated with LPS + nigericin (Pan et al., 2021) (Figure 3B). The expression of 2-E+2-3a in both cell systems, 293T (Figure 3C) and THP1 macrophages (Figure 3D-F), resulted in enhanced secretion of NLRP3-activated IL-1 $\beta$ secretion.

We then confirmed that 2-E+2-3a expression activates the NLRP3-I and IL-1 $\beta$ secretion via increased mROS production. 293T cells expressing the inflammasome proteins (Figure 3C) and LPS-nigericin treated THP1 macrophages (Figure 3D\&E) were treated with mitochondrially targeted antioxidants, transformation with mCAT or treatment with MnTBAP. Both mCAT expression and MnTBAP treatment impaired IL-1 $\beta$ secretion.

We then determined if mROS activation of the NLRP3-I was mediated by release of an oxidized mitochondrial component via the mtPTP, which has been conjectured but not proven. We treated 2-E+2-3a transformed THP1 macrophages with the specific mtPTP inhibitor N-methyl-4-isoleucine-cyclosporin (NIM811). NIM811 blocks the mtPTP by binding to cyclophilin D, analogous to cyclosporin A (CsA) but without calcineurin inactivation (Dittmar et al., 2021; Tóth et al., 2019; Zhang et al., 2020). NIM811 treatment suppressed the secretion of IL-1 $\beta$ following LPS + nigericin activation of the THP1 macrophages demonstrating for the first time the mIPTP is the route by which an oxidized mitochondrial factor reaches the NRLP3-I (Figure 3F).

Thus, we have demonstrated that co-expression of $2-\mathrm{E}+2-3 \mathrm{a}$ enhances $\mathrm{Ca}^{++}$leakage into the cytosol, increasing levels of cytosolic and mitochondrial $\mathrm{Ca}^{++}$. This $2-\mathrm{E}+2-3 \mathrm{a}$ mediated increase in mitochondrial $\mathrm{Ca}^{++}$ induces the production of mROS, which in turn activates the NLRP3-I, via mIPTP transport of an oxidized mitochondrial product, stimulating the secretion of IL-1 $\beta$. Increasing mitochondrial antioxidant defenses through treatment with the pharmacological mROS scavenger MnTBAP, or genetic expression of mCAT, detoxifies 2- 
$\mathrm{E}+2-3 \mathrm{a}$ induced $\mathrm{mROS}$ and blocks activation of the NLRP3-I. Together these findings reveal that the mechanism by which 2-E+2-3a engage the NLRP3-I is via viroporin manipulation of mitochondrial physiology.

\section{DISCUSSION}

Because of the importance that activation of the inflammasome by SARS-CoV-2 has on the severity of COVID19, we set out to define the physiological process by which the virus activates the NLRP3-I in hopes of identifying drug targets to mitigate the cytokine storm. We found that the viroporins $2-E+2-3 a$ were central to the activation of the NLRP3-I and this occurred through a mitochondrial innate immunity signal transduction pathway. Expression of viroporins 2-E+2-3a results in the release of $\mathrm{ER}$ and extracellular $\mathrm{Ca}^{++}$into the cytosol where the $\mathrm{Ca}^{++}$is taken up by the mitochondrion via the mitochondrial calcium uniporter. The mitochondrial $\mathrm{Ca}^{++}$activates the tricarboxylic acid cycle dehydrogenases generating excess NADH (Denton, 2009). This overloads the electron transport chain producing increased mROS. The increased mROS oxidizes a mitochondrial factor that is released through the MIPTP to bind and activate the NLRP3-I.

While our experiments, did not directly identify the mitochondrial factor released through the mtPTP, other recent studies have shown that this factor is oxidized mtDNA which is a ligand of NLRP3-I (Xian et al., 2021; Zhong et al., 2018). Thus, we complete the mitochondrial innate immune activation pathway by showing that release of Ox-mtDNA is via the mtPTP.

Demonstration that SARS-CoV-2 activated the inflammasome via the mitochondria provides new approaches to mitigating the severity of the cytokine storm. Previous studies have indicated that generalized antioxidants such as N-acetyl cysteine (Garozzo et al., 2007; Geiler et al., 2010; Ghezzi and Ungheri, 2004), glutathione (Cai et al., 2003; Nencioni et al., 2003), and catalase (Shi et al., 2014; Shi et al., 2010) can reduce viral propagation and pathology. Our data extend these observations by indicating that the therapeutic potential of drugs will be enhanced if they are mitochondrially targeted antioxidants such as MnTBAP (Melov et al., 1998; Tong et al., 2007), EUK-8 and EUK-134 (Melov et al., 2001) and or inhibitors of the mtPTP such as NIM811 (Dittmar et al., 2021; Tóth et al., 2019; Zhang et al., 2020).

\section{Limitations of the Study.}

A unique feature of this research is the discovery that SARS-CoV-2 viroporins active the inflammasome via the mitochondrion through elevated mitochondrial $\mathrm{Ca}^{++}$and $\mathrm{mROS}$ and the mtPTP. However, we have not identified this released mitochondrial oxidized product. Rather, we relied of the publications of others implicating OxmtDNA.

\section{ACKNOWLEDGMENTS}

The authors thank Dr. Bruce Beutler for gifts of expression plasmids and Dr. Cristina Mammucari (Department of Biomedical Sciences, University of Padua, 35131 Padua, Italy) and Dr. Kevin Foskett (Dept Cell and Developmental Biology, University of Pennsylvania, 19104 Philadelphia, Pennsylvania) for gifting MCUi11, which they purchased from AKos Consulting \& Solutions $\mathrm{GmbH}$. This work was supported by DOD grant W81XWH-21-1-0128 awarded to D.C.W.

\section{AUTHOR CONTRIBUTIONS}

Conceptualization: J.W.G., D.C.W.; Methodology: J.W.G., P.P, D.C.W., A.A., D.M.; Literature and concept integration: J.W.G., P.P, D.C.W., A.A., D.M.; Formal Analysis: J.W.G., A.A., D.M., D.C.W.; Writing - Original Draft: J.W.G.; Investigation: J.W.G., A.A., D.M., D.C.W., T.L.; Sample Collection: J.W.G., T.L.; Writing - Review \& Editing: J.W.G., D.C.W., A.A., D.M.; Visualization: J.W.G., D.C.W., A.A., D.M.; Supervision: J.W.G., P.P, D.C.W., A.A., D.M.; Funding Acquisition: D.C.W.

\section{DECLARATION OF INTERESTS}

D.C.W. serves of the advisory boards of Plano Therapeutics, Medical Excellent Capital, and has a grant from March Therapeutics. 


\section{MAIN FIGURE TITLES AND LEGENDS}

Figure 1. Expression of 2-E+2-3a induces mROS production by elevating mitochondrial Ca ${ }^{++}$levels. A-B) Schematics of our LV-EV and LV-E3a vectors. C) 293T cells were transduced with LV-EV or LV-E3a, and samples analyzed by immunoblot with a mouse monoclonal antibody against FLAG-Tag, to detect the FLAGtagged 2-E+2-3a viroporins. GAPDH was a loading control. D-F) $24 \mathrm{hrs}$ post-transduction cells were stained with Fura-Red to measure cytosolic $\mathrm{Ca}^{++}$levels, G-I) Rhod2 \& Mitotracker Deep Red (MTDR) to measure mitochondrial $\mathrm{Ca}^{++}$levels, or K-M) MitoSOX and MTDR to measure mROS levels via E-F. H-I, L-M) data from confocal microscopy or $\mathbf{D}, \mathbf{G}, \mathbf{K}$ ) data from plate reader assays. J, N) 24 hrs post-transduction with LV-EV or LVE3a 293T cells were treated with or without $10 \mu \mathrm{M}$ MCUi11, and then $2.5 \mu \mathrm{M} \mathrm{TG}$ and stained with J) Rhod2 to measure mitochondrial $\mathrm{Ca}^{++}$levels, or $\mathrm{N}$ ) MitoSOX to measure mROS levels by plate reader assays. Scale bar $=30 \mu \mathrm{m}$. Error bars represent SEM from 3 independent experiments; statistically significant data is indicated with asterisks $\left(^{*}\right)$.

Figure 2. Expression of mCAT or treatment with the mROS scavenger MnTBAP antioxidant defenses blocks 2-E+2-3a induced mROS. A-B) Schematics of $p$-EV and p-mCAT vectors. C) Catalase assay through cleavage of $\mathrm{H}_{2} \mathrm{O}_{2}$ in 293T cell lysates collected $24 \mathrm{hrs}$ post-transfection with p-EV or p-mCAT. D-I) 24 hrs posttransfection levels of mROS assessed using MitoSOX and MTDR fluorescence 293T cells transduced with LVEV or LV-E3a and transfected $\mathbf{D}, \mathbf{E}$, and $\mathbf{H}$ ) with p-mCAT or its respective control p-EV or $\mathbf{F}, \mathbf{G}$, and I) cultured in the presence or absence of $50 \mu \mathrm{M}$ MnTBAP, DMSO used as a negative control. MTDR fluorescence was used to normalize for mitochondrial content with mROS expressed as the ratio of MitoSOX/MTDR, by D, F) confocal microscopy or $\mathbf{E}, \mathbf{G}$ ) plate reader assays $\mathbf{H}, \mathbf{I})$ Representative images of MitoSOX-stained cells. Scale bar $=30$ $\mu \mathrm{m}$. Error bars represent SEM from 3 independent experiments; statistically significant data is indicated with asterisks $\left({ }^{*}\right)$.

Figure 3. mROS and the mtPTP are required for activation of the NLRP3-I by the 2-E+2-3a viroporins. AB) Experimental design used to assess NLRP3-activated by IL-1 $\beta$ in cell-free supernatants quantified by ELISA. A) $293 \mathrm{~T}$ cells with an NLRP3-I reconstitution system (NLRP3, ASC, pro-CASP1, pro-IL-1 $\beta$ ) and B) THP1 differentiated into macrophages and primed with LPS + nigericin. C) 293T cells transfected with LV-EV or LVE3a were transformed the NLRP3-I plasmids and p-mCAT or its control plasmid p-EV, or cultured in the presence or absence of $50 \mu \mathrm{M}$ MnTBAP. D, F) THP1 cells were transduced with LV-EV or LV-E3a, differentiated into macrophages, treated with LPS and nigericin, and treated with $100 \mu \mathrm{M}$ MnTBAP or $10 \mu \mathrm{M}$ NIM811, the supernatants analyzed for IL-1 $\beta$ by ELISA. E) THP1 cells stably expressing LV-mCAT or control were infected with LV-EV or LV-E3a, differentiated into macrophages, and supernatant IL-1 $\beta$ levels determined via ELISA. Error bars represent SEM from 3 independent experiments; statistically significant data is indicated with asterisks $\left({ }^{*}\right)$.

\section{MAIN TABLES AND LEGENDS Non-Applicable}

\section{STAR $\star M E T H O D S$}

\section{RESOURCE AVAILABILITY}

\section{Lead Contact.}

Further information and requests for resources and reagents should be directed to and will be fulfilled by the Lead Contact, \& Douglas C. Wallace (WallaceD1@chop.edu).

\section{Materials Availability.}

This study did not generate new unique reagents.

\section{Data and Code Availability.}



available under aCC-BY-NC-ND 4.0 International license.

This study did not generate any unique datasets. All data is included in the manuscript or supplementary file.

\section{EXPERIMENTAL MODEL AND SUBJECT DETAILS}

\section{Cells, Infections, \& Reagents.}

293T \& THP1 cells were obtained from the American Type Culture Collection (ATCC). Cells were grown at $37^{\circ} \mathrm{C}$ with an atmosphere of $98 \%$ humidity and $5 \% \mathrm{CO}_{2}$. $293 \mathrm{~T}$ cells were maintained in Dulbecco's modified Eagle's medium + GlutaMAXTM supplement with pyruvate (GIBCO), $1 \%$ non-essential amino acids (SIGMA), and 10\% fetal bovine serum (FBS) (Takara Bio). THP1 cells were grown in RPMI 1640 Medium (GIBCO) supplemented with $10 \%$ FBS (Takara Bio). 293T cells were infected (MOI 4) as previously described (Potluri et al., 2016). THP1 cells were infected (MOI 8) with the addition of $5 \mu \mathrm{g} / \mathrm{ml}$ polybrene (VectorBuilder) and spininoculated at $700 \times \mathrm{g}$ for $25 \mathrm{~min}$.

\section{Plasmids, Viral Vectors, THP1 stable-transformants.}

Plasmid Vectors. To express the components of the NLRP3-inflammasome (NLFP3-I), we utilized four plasmids expressing mouse NLRP3 (pcDNA3-N-Flag-NLRP3, Addgene plasmid \# 75127), ASC (pcDNA3-N-Flag-ASC1, Addgene plasmid \# 75134), CASP1 (pcDNA3-N-Flag-Caspase-1, Addgene plasmid \# 75128) and pro-IL-1B (pCMV-pro-II1b-C-Flag, Addgene plasmid \# 75131), The use and construction of the NLRP3-I expression plasmids were previously described (Shi et al., 2016).

The plasmid vector used to express mitochondrial-targeted catalase (mCAT) and its respective control vector were p-mCAT (VectorBuilder ID VB170403-1078nzg) and p-EV (VectorBuilder ID VB210726-1273jte), vectorbuilder.com. The $p$-mCAT transgene cassette is transcribed from the 212 nucleotide elongation factor $\alpha 1$ "short" (EFS) promoter. The EFS promoter transcribes a polycistronic transcript encoding EGFP (enhanced green fluorescent protein), a self-cleaving 2A peptide site, followed by mCAT, terminated by a simian virus 40 (SV40) late polyA sequence. $\mathrm{p}-\mathrm{EV}$ is identical to the $\mathrm{p}$-mCAT construct, except lacking the mCAT sequence.

Lentiviral Vectors. The lentiviral vector used to co-express 2-E+2-3a was LV-E3a (Vectorbuilder ID VB2101121153ufz) and its respective control vector LV-EV (Vectorbuilder ID VB210112-1153ufz). The LV-E3a vector contains the cytomegalovirus (CMV) promoter, the 2-E+2-3a viroporins obtained from Gordon et al. 2020 (Gordon et al., 2020b) separated by a 2A peptide site, and terminated by a SV40 late polyA sequence cloned into the LV-EV vector. The viroporins were modified by addindg anATG codon 5' and three N-terminal FLAGtags were added to the $3^{\prime}$ end of each viral protein, and transcribed from the the proteins. LV-EV is an empty vector.

The lentiviral vector expressing our mCAT and its respective control vector, LV-mCAT (VB2109091242kdf) and LV-EV(mCAT) (VB900122-0484ubz) were constructed and packaged by VectorBuilder. The LVmCAT vector includes the EFS promoter, EGFP, 2A peptide site, mCAT, , and SV40 late polyA sequence. The LV-EV (mCAT) control vector lacks EGFP and mCAT.

THP1 mCAT stable-transformants. THP1 cells were transduced with LV-mCAT or empty vector and selected with puromycin. Expression of mCAT was Ivalidated stable-transformants by EGFP fluorescence.

\section{METHOD DETAILS}

\section{Cell Staining.}

293T cells were plated at a density of $45 \times 10^{3}$ in 96 -well $0.2 \%$ gelatin-coated (ScienCell) glass-bottom plates with high-performance \#1.5 mm cover glass (Cellvis). Twenty-four hours post-plating, sub-confluent monolayers of 293T cells were transduced with LV-EV or LV-E3a. Twenty-four hours post-transduction, cells were washed two times with phosphate-buffered saline (PBS), then stained. For determination of mROS levels, cells were co-stained with $3 \mu \mathrm{M}$ MitoSOX ${ }^{\mathrm{TM}}$ Red (MitoSOX, mitochondrial superoxide indicator) and $50 \mathrm{nM}$ MitoTracker ${ }^{\mathrm{TM}}$ Deep Red FM (MTDR) for $30 \mathrm{~min}$ at $37^{\circ} \mathrm{C}$. For assaying mROS levels after treatment with Thapsigargin (TG) using the plate reader, cells were stained with $3 \mu \mathrm{M}$ MitoSOX for $30 \mathrm{~min}$ at $37^{\circ} \mathrm{C}$. To quantify mROS after staining, cells were washed three times in PBS, maintained in FluoroBrite ${ }^{\mathrm{TM}}$ DMEM (GIBCO) supplemented with $12.5 \mathrm{mM} \mathrm{HEPES} \mathrm{(SIGMA)} \mathrm{and} \mathrm{1 \%} \mathrm{non-essential} \mathrm{amino} \mathrm{acids} \mathrm{(SIGMA),} \mathrm{and} \mathrm{the} \mathrm{fluorescence}$ measures 
To determine cytosolic $\mathrm{Ca}^{++}$levels, cells were washed three times with Tyrode's Salts (Sigma-Aldrich), stained for 40 min with $2 \mu \mathrm{M}$ Fura Red ${ }^{\mathrm{TM}}$, acetoxymethyl ester (AM), cell-permeant (Fura-Red) in $0.02 \%$ pluronic F127 (Pluronic® F-127) detergent. To determine mitochondrial $\mathrm{Ca}^{++}$levels, cells were washed three times with Tyrode's Salts, stained for 40 min with $10 \mu \mathrm{M}$ Rhod-2, AM, cell-permeant (Rhod2) and $50 \mathrm{nM}$ MTDR in 0.02\% pluronic F127. After staining cells were washed three times and maintained in Tyrode's Salts and florescence measured. To determine mitochondrial $\mathrm{Ca}^{++}$levels after treating with TG, cells were stained for 40 min with 10 $\mu \mathrm{M}$ Rhod2, washed three times and maintained in Tyrode's Salts, and florescence measured.

\section{SpectraMax Plate Reader Assay.}

Measurement of mROS and cytosolic and mitochondrial Ca ${ }^{++}$levels. After staining cells (see "Cell Staining"), mean fluorescence was assessed using the SpectraMax $\AA$ Paradigm $\AA$ Multi-mode Detection Platform, equipped with a Tunable Wavelength (TUNE) Detection Cartridge (Molecular Devices). was quantified by MitoSOX fluorescence (ex:540 nm, em:590 nm) and MTDR (ex:633 nm, em:680 nm) and MitoSOX/MTDR calculated. Rhod2 fluorescence for mitochondrial Ca ${ }^{++}$level (ex:540 nm, em:590 nm) and MTDR (ex:633 nm, em:680 nm), and Rhod2/MTDR calculated. Fura-Red fluorescence for cytosolic bound-Ca ${ }^{++}$level (ex:405 nm, em:637 nm) and unbound-Ca ${ }^{++}$(ex:514 nm, em:672 nm) state. The ratio of bound-Ca $\mathrm{Ca}^{++} /$unbound-Ca ${ }^{++}$was calculated.

Measurement of mitochondrial $\mathrm{Ca}^{++}$and mROS after treating with TG. After staining cells with Rhod2 or MitoSOX ("Cell Staining"), cells were treated for $10 \mathrm{~min}$ with or without $10 \mu \mathrm{M}$ mitochondria channel uniporter inhibitor 11 (MCUi11), Dimethylsulfoxide (DMSO) as a negative control. Mitochondrial $\mathrm{Ca}^{++}$determined from Rhod2 (ex:540 $\mathrm{nm}$, em:590 nm) and mROS from MitoSOX (ex:540 nm, em:590 nm). Cells were tthen treated with $2.5 \mu \mathrm{M}$ TG and changes in mitochondrial $\mathrm{Ca}^{++}$or mROS recorded every 15 seconds for 180 seconds. Relative change in mitochondrial $\mathrm{Ca}^{++}$was calculated by dividing the average change in Rhod2 fluorescence after treatment with TG by Rhod2 fluorescence before treatment with TG. Relative change in mROS levels was calculated by dividing the average change in MitoSOX fluorescence after treatment with TG by MitoSOX fluorescence before treatment with TG.

\section{Confocal Microscopy.}

Live-cell imaging was performed using a Zeiss 710 LSM confocal microscope with an environmental chamber maintained at $37^{\circ} \mathrm{C}$ and $5 \%$ CO2. Laser lines used: diode $405 \mathrm{~nm}$, Argon $514 \mathrm{~nm}$, HeNe lasers $543 \mathrm{~nm}$ and $633 \mathrm{~nm}$ excitation wavelengths. Fluorescence quantified using the Zeiss $710 \mathrm{LSM}$ confocal microscope was analyzed using ImageJ. Fluorescence intensities of stained cells were normalized to the unstained negative cells. A minimum of 60 images was taken for each condition across at least three independent experiments.

\section{Catalase Assay.}

Catalase activity was assessed through cleavage of $\mathrm{H}_{2} \mathrm{O}_{2}$ in 293T cell lysates collected twenty-four hours post-transfection with p-mCAT or p-EV, using a Catalysis Activity Kit (Abcam, ab83464).

\section{Western blot analysis.}

Twenty-four hours post-transduction, cells were washed once with cold PBS and then lysed with $2.5 \% \mathrm{n}$ Dodecyl-B-D-Maltoside in 20 mM HEPES (pH 7.4), 50 mM ßglycerophosphate, 2 mM EGTA, 10\% (v/v) glycerol, and $0.01 \%$ Bromophenol blue. Lysates were electrophoresed on 4 to $12 \%$, Bis-Tris gels (Invitrogen) SDSpolyacrylamide NuPAGE ${ }^{\text {TM }}$ gels Gels were ransferred to a nitrocellulose membrane by the iBlot Gel Transfer System (Invitrogen), membranes blocked for one hour in $5 \%$ nonfat milk in $25 \mathrm{mM}$ Tris- $\mathrm{HCl}, 150 \mathrm{mM} \mathrm{NaCl}, 0.1 \%$ Tween 20 (TBST buffer and incubated overnight at $4^{\circ} \mathrm{C}$ with shaking in primary antibody diluted 1:1000 in TBST. Membranes were then washed three times with TBST and incubated with Alexa Fluor-conjugated secondary antibodies for one hour at room temperature. Protein levels were quantified using the Odyssey imaging system (LiCOR Biosciences) using. GAPDH as a loading control.

\section{Detection of secreted IL-1 $\beta$ in 293T cells with a reconstituted NLRP3-I and THP1 macrophages.}

293T cells were plated at a density of $100 \times 10^{3}$ in 24-well plates. Twenty-four hours post-plating subconfluent monolayers of 293 T cells were infected with LV-EV or LV-E3a. Six hours post-infection cells were cotransfected using the TransIT-X2® Dynamic Delivery System (Mirus Bio) with the plasmids encoding the components of the NLRP3-I (Shi et al., 2016), followed by p-mCAT or p-EV transduction. Twenty-four hours post-transfection, cells were washed two times with PBS, then cultured with or without the addition of 50 uM 
MnTBAP. Cell lysates and culture supernatants were collected twelve hours post-treatment andcentrifuged to remove cell debris. Supernatant IL-1 $\beta$ was quantified by ELISA (Abcam, ab197742).

THP-1 cells were plated at a density of $100 \times 10^{3}$ in 96-well plates. THP1 cells were infected with LV-EV or LV-E3a. Six hours post-infection, THP1 cells were differentiated into macrophages with $50 \mathrm{ng} / \mathrm{ml} \mathrm{Phorbol} 12-$ myristate 13-acetate (PMA) overnight. After differentiation, cells were washed two times with PBS, and fresh media was added with addition of $100 \mu \mathrm{M}$ MnTBAP or $10 \mu \mathrm{M} \mathrm{N}$-methyl-4-isoleucine-cyclosporin (NIM811). Nine hours post-treatment with MnTBAP or one hour post-treatment with NIM811 THP1 macrophages were stimulated with $100 \mathrm{ng} / \mathrm{ml}$ Lipopolysaccharides (LPS) and $2.5 \mathrm{mM}$ of nigericin for nine hrousSupernatants were collected, centrifuged, and the amount of supernatants IL-1 $\beta$ measured by ELISA (Abcam, ab46052). THP1 cells stably expressing LV-mCAT or control vector, were infected with LV-EV or LV-E3a, and 6 hours post-infection the THP1 cells were differentiated into macrophages, and supernatant IL-1 $\beta$ quantified via ELISA.

\section{QUANTIFICATION AND STATISTICAL ANALYSIS}

\section{Statistical analysis}

One-way ANOVA was performed for statistical differences between three or more groups, followed by a post hoc Tukey's HSD test to test for statistical differences. For studies that require a quantitative evaluation between two groups, statistical significance was determined using unpaired two-tail student's t-test. All data are reported as mean \pm standard error of the mean (SEM). All statistical analysis was done on GraphPad Prism 9.01. For student's t-test ${ }^{*}=p<$ value $0.05,{ }^{\star *}=p<$ value $0.01,{ }^{\star * *}=p<$ value $0.001,{ }^{\star * \star *}=p<$ value 0.0001 ).

\section{SUPPLEMENTAL VIDEO, DATA, AND EXCEL TABLE TITLE AND LEGENDS Non-Applicable}

\section{REFERENCES}

1. Ajaz, S., McPhail, M.J., Singh, K.K., Mujib, S., Trovato, F.M., Napoli, S., and Agarwal, K. (2021). Mitochondrial metabolic manipulation by SARS-CoV-2 in peripheral blood mononuclear cells of patients with COVID-19. American Journal of Physiology. Cell Physiology 320, C57-C65.

2. Bagur, R., and Hajnóczky, G. (2017). Intracellular $\mathrm{Ca}(2+)$ sensing: its role in calcium homeostasis and signaling. Mol. Cell. 66, 780-788.

3. Broz, P., and Dixit, V.M. (2016). Inflammasomes: mechanism of assembly, regulation and signalling. Nat Rev Immunol 16, 407-420.

4. Cai, J., Chen, Y., Seth, S., Furukawa, S., Compans, R.W., and Jones, D.P. (2003). Inhibition of influenza infection by glutathione. Free Radical Biology and Medicine 34, 928-936.

5. Chen, G., Wu, D., Guo, W., Cao, Y., Huang, D., Wang, H., Wang, T., Zhang, X., Chen, H., Yu, H., et al. (2020). Clinical and immunological features of severe and moderate coronavirus disease 2019. J. Clin. Invest. 130, 2620-2629.

6. Chen, I.Y., Moriyama, M., Chang, M.F., and Ichinohe, T. (2019). Severe acute respiratory syndrome coronavirus viroporin 3a activates the NLRP3 inflammasome. Frontiers in Microbiology 10, 50.

7. Chi, Y., Ge, Y., Wu, B., Zhang, W., Wu, T., Wen, T., Liu, J., Guo, X., Huang, C., Jiao, Y., et al. (2020). Serum cytokine and chemokine profile in relation to the severity of coronavirus disease 2019 in China. J. Infect. Dis. 222, 746-754.

8. Csordás, G., Weaver, D., and Hajnóczky, G. (2018). Endoplasmic reticulum-mitochondrial contactology: structure and signaling functions. Trends Cell Biol. 28, 523-540. 
bioRxiv preprint doi: https://doi.org/10.1101/2022.02.19.481139; this version posted February $22,2022$. The copyright holder for this preprint (which was not certified by peer review) is the author/funder, who has granted bioRxiv a license to display the preprint in perpetuity. It is made available under aCC-BY-NC-ND 4.0 International license.

9. Denton, R.M. (2009). Regulation of mitochondrial dehydrogenases by calcium ions. Biochim. Biophys. Acta. 1787, 1309-1316.

10. Di Marco, G., Vallese, F., Jourde, B., Bergsdorf, C., Sturlese, M., De Mario, A., Techer-Etienne, V., Haasen, D., Oberhauser, B., Schleeger, S., et al. (2020). A high-throughput screening identifies MICU1 targeting compounds. Cell Rep 30, 2321-2331.e2326.

11. Dittmar, M., Lee, J.S., Whig, K., Segrist, E., Li, M., Kamalia, B., Castellana, L., Ayyanathan, K., CardenasDiaz, F.L., Morrisey, E.E., et al. (2021). Drug repurposing screens reveal cell-type-specific entry pathways and FDA-approved drugs active against SARS-Cov-2. Cell Rep 35, 108959.

12. Dong, E., Du, H., and Gardner, L. (2020). An interactive web-based dashboard to track COVID-19 in real time. Lancet Infect. Dis. 20, 533-534.

13. Ferreira, A.C., Soares, V.C., de Azevedo-Quintanilha, I.G., Dias, S., Fintelman-Rodrigues, N., Sacramento, C.Q., Mattos, M., de Freitas, C.S., Temerozo, J.R., Teixeira, L., et al. (2021). SARS-CoV-2 engages inflammasome and pyroptosis in human primary monocytes. Cell Death Discovery 7, 43.

14. Garozzo, A., Tempera, G., Ungheri, D., Timpanaro, R., and Castro, A. (2007). N-acetylcysteine synergizes with oseltamivir in protecting mice from lethal influenza infection. Int. J. Immunopathol. Pharmacol. 20, 349354.

15. Geiler, J., Michaelis, M., Naczk, P., Leutz, A., Langer, K., Doerr, H.W., and Cinatl, J. (2010). N-acetyl-Lcysteine (NAC) inhibits virus replication and expression of pro-inflammatory molecules in A549 cells infected with highly pathogenic H5N1 influenza A virus. Biochem. Pharmacol. 79, 413-420.

16. Ghezzi, P., and Ungheri, D. (2004). Synergistic combination of $\mathrm{N}$-acetylcysteine and ribavirin to protect from lethal influenza viral infection in a mouse model. Int. J. Immunopathol. Pharmacol. 17, 99-102.

17. Gordon, D.E., Hiatt, J., Bouhaddou, M., Rezelj, V.V., Ulferts, S., Braberg, H., Jureka, A.S., Obernier, K., Guo, J.Z., Batra, J., et al. (2020a). Comparative host-coronavirus protein interaction networks reveal pan-viral disease mechanisms. Science 370, eabe9403.

18. Gordon, D.E., Jang, G.M., Bouhaddou, M., Xu, J., Obernier, K., White, K.M., O'Meara, M.J., Rezelj, V.V., Guo, J.Z., Swaney, D.L., et al. (2020b). A SARS-CoV-2 protein interaction map reveals targets for drug repurposing. Nature 583, 459-468.

19. Han, Y., Zhang, H., Mu, S., Wei, W., Jin, C., Tong, C., Song, Z., Zha, Y., Xue, Y., and Gu, G. (2020). Lactate dehydrogenase, an independent risk factor of severe COVID-19 patients: a retrospective and observational study. Aging 12, 11245-11258.

20. Hover, S., Foster, B., Barr, J.N., and Mankouri, J. (2017). Viral dependence on cellular ion channels - an emerging anti-viral target? J Gen Virol 98, 345-351.

21. Kern, D.M., Sorum, B., Mali, S.S., Hoel, C.M., Sridharan, S., Remis, J.P., Toso, D.B., Kotecha, A., Bautista, D.M., and Brohawn, S.G. (2021). Cryo-EM structure of SARS-CoV-2 ORF3a in lipid nanodiscs. Nat. Struct. Mol. Biol. 28, 573-582.

22. Liu, Z., Chen, O., Wall, J.B.J., Zheng, M., Zhou, Y., Wang, L., Ruth Vaseghi, H., Qian, L., and Liu, J. (2017). Systematic comparison of 2A peptides for cloning multi-genes in a polycistronic vector. Sci. Rep. 7, 2193.

23. Lucas, C., Wong, P., Klein, J., Castro, T.B.R., Silva, J., Sundaram, M., Ellingson, M.K., Mao, T., Oh, J.E., Israelow, B., et al. (2020). Longitudinal analyses reveal immunological misfiring in severe COVID-19. Nature 584, 463-469. 
24. Mandala, V.S., McKay, M.J., Shcherbakov, A.A., Dregni, A.J., Kolocouris, A., and Hong, M. (2020). Structure and drug binding of the SARS-CoV-2 envelope protein transmembrane domain in lipid bilayers. Nat. Struct. Mol. Biol. 27, 1202-1208.

25. Márta, K., Hasan, P., Rodríguez-Prados, M., Paillard, M., and Hajnóczky, G. (2021). Pharmacological inhibition of the mitochondrial $\mathrm{Ca}(2+)$ uniporter: relevance for pathophysiology and human therapy. Journal of molecular and cellular cardiology 151, 135-144.

26. Melov, S., Doctrow, S.R., Schneider, J.A., Haberson, J., Patel, M., Coskun, P.E., Huffman, K., Wallace, D.C., and Malfroy, B. (2001). Lifespan extension and rescue of spongiform encephalopathy in superoxide dismutase 2 nullizygous mice treated with superoxide dismutase-catalase mimetics. Journal of Neuroscience $21,8348-8353$.

27. Melov, S., Schneider, J.A., Day, B.J., Hinerfeld, D., Coskun, P., Mirra, S.S., Crapo, J.D., and Wallace, D.C. (1998). A novel neurological phenotype in mice lacking mitochondrial manganese superoxide dismutase. Nat. Genet. 18, 159-163.

28. Minakshi, R., and Padhan, K. (2014). The YXXФ motif within the severe acute respiratory syndrome coronavirus (SARS-CoV) 3a protein is crucial for its intracellular transport. Virol. J. 11, 75.

29. Nencioni, L., luvara, A., Aquilano, K., Ciriolo, M.R., Cozzolino, F., Rotilio, G., Garaci, E., and Palamara, A.T. (2003). Influenza A virus replication is dependent on an antioxidant pathway that involves GSH and Bcl-2. FASEB Journal 17, 758-760.

30. Nieto-Torres, J.L., DeDiego, M.L., Verdiá-Báguena, C., Jimenez-Guardeño, J.M., Regla-Nava, J.A., Fernandez-Delgado, R., Castaño-Rodriguez, C., Alcaraz, A., Torres, J., Aguilella, V.M., et al. (2014). Severe acute respiratory syndrome coronavirus envelope protein ion channel activity promotes virus fitness and pathogenesis. PLoS Pathog. 10, e1004077.

31. Nieto-Torres, J.L., Verdiá-Báguena, C., Jimenez-Guardeño, J.M., Regla-Nava, J.A., Castaño-Rodriguez, C., Fernandez-Delgado, R., Torres, J., Aguilella, V.M., and Enjuanes, L. (2015). Severe acute respiratory syndrome coronavirus $\mathrm{E}$ protein transports calcium ions and activates the NLRP3 inflammasome. Virology 485, 330-339.

32. Nieva, J.L., Madan, V., and Carrasco, L. (2012). Viroporins: structure and biological functions. Nat. Rev. Microbiol. 10, 563-574.

33. Pan, P., Shen, M., Yu, Z., Ge, W., Chen, K., Tian, M., Xiao, F., Wang, Z., Wang, J., Jia, Y., et al. (2021). SARS-CoV-2 N protein promotes NLRP3 inflammasome activation to induce hyperinflammation. Nat. Commun. 12, 4664.

34. Potluri, P., Procaccio, V., Scheffler, I.E., and Wallace, D.C. (2016). High throughput gene complementation screening permits identification of a mammalian mitochondrial protein synthesis $(\rho-)$ mutant. Biochim. Biophys. Acta. 1857, 1336-1343.

35. Qu, Y., Wang, X., Zhu, Y., Wang, W., Wang, Y., Hu, G., Liu, C., Li, J., Ren, S., Xiao, M.Z.X., et al. (2021). ORF3a-mediated incomplete autophagy facilitates severe acute respiratory syndrome coronavirus-2 replication. Frontiers in cell and developmental biology 9, 716208.

36. Rodrigues, T.S., de Sá, K.S.G., Ishimoto, A.Y., Becerra, A., Oliveira, S., Almeida, L., Gonçalves, A.V., Perucello, D.B., Andrade, W.A., Castro, R., et al. (2021). Inflammasomes are activated in response to SARSCoV-2 infection and are associated with COVID-19 severity in patients. The Journal of Experimental Medicine 218, e20201707. 
37. Schriner, S.E., Linford, N.J., Martin, G.M., Treuting, P., Ogburn, C.E., Emond, M., Coskun, P.E., Ladiges, W., Wolf, N., Van Remmen, H., et al. (2005). Extension of murine life span by overexpression of catalase targeted to mitochondria. Science 308, 1909-1911.

38. Shi, H., Wang, Y., Li, X., Zhan, X., Tang, M., Fina, M., Su, L., Pratt, D., Bu, C.H., Hildebrand, S., et al. (2016). NLRP3 activation and mitosis are mutually exclusive events coordinated by NEK7, a new inflammasome component. Nat. Immunol. 17, 250-258.

39. Shi, X., Shi, Z., Huang, H., Zhu, H., Zhou, P., Zhu, H., and Ju, D. (2014). Ability of recombinant human catalase to suppress inflammation of the murine lung induced by influenza A. Inflammation 37, 809-817.

40. Shi, X.L., Shi, Z.H., Huang, H., Zhu, H.G., Zhou, P., and Ju, D. (2010). Therapeutic effect of recombinant human catalase on H1N1 influenza-induced pneumonia in mice. Inflammation 33, 166-172.

41. Siu, K.L., Yuen, K.S., Castano-Rodriguez, C., Ye, Z.W., Yeung, M.L., Fung, S.Y., Yuan, S., Chan, C.P., Yuen, K.Y., Enjuanes, L., et al. (2019). Severe acute respiratory syndrome coronavirus ORF3a protein activates the NLRP3 inflammasome by promoting TRAF3-dependent ubiquitination of ASC. FASEB Journal $33,8865-8877$.

42. Szymczak-Workman, A.L., Vignali, K.M., and Vignali, D.A. (2012). Verification of 2A peptide cleavage. Cold Spring Harb Protoc 2012, 255-257.

43. Tong, J., Schriner, S.E., McCleary, D., Day, B.J., and Wallace, D.C. (2007). Life extension through neurofibromin mitochondrial regulation and antioxidant therapy for Neurofibromatosis-1 in Drosophila melanogaster. Nat. Genet. 39, 476-485.

44. Tóth, E., Maléth, J., Závogyán, N., Fanczal, J., Grassalkovich, A., Erdős, R., Pallagi, P., Horváth, G., Tretter, L., Bálint, E.R., et al. (2019). Novel mitochondrial transition pore inhibitor N-methyl-4-isoleucine cyclosporin is a new therapeutic option in acute pancreatitis. The Journal of Physiology 597, 5879-5898.

45. Verdiá-Báguena, C., Aguilella, V.M., Queralt-Martín, M., and Alcaraz, A. (2021). Transport mechanisms of SARS-CoV-E viroporin in calcium solutions: Llipid-dependent anomalous mole fraction effect and regulation of pore conductance. Biochimica et biophysica acta. Biomembranes 1863, 183590.

46. Verdiá-Báguena, C., Nieto-Torres, J.L., Alcaraz, A., DeDiego, M.L., Torres, J., Aguilella, V.M., and Enjuanes, L. (2012). Coronavirus $E$ protein forms ion channels with functionally and structurally-involved membrane lipids. Virology 432, 485-494.

47. Wen, W., Su, W., Tang, H., Le, W., Zhang, X., Zheng, Y., Liu, X., Xie, L., Li, J., Ye, J., et al. (2020). Immune cell profiling of COVID-19 patients in the recovery stage by single-cell sequencing. Cell Discov. 6, 31.

48. West, A.P., and Shadel, G.S. (2017). Mitochondrial DNA in innate immune responses and inflammatory pathology. Nat Rev Immunol 17, 363-375.

49. Xia, B., Shen, X., He, Y., Pan, X., Liu, F.L., Wang, Y., Yang, F., Fang, S., Wu, Y., Duan, Z., et al. (2021). SARS-CoV-2 envelope protein causes acute respiratory distress syndrome (ARDS)-like pathological damages and constitutes an antiviral target. Cell Res. 31, 847-860.

50. Xian, H., Liu, Y., Rundberg Nilsson, A., Gatchalian, R., Crother, T.R., Tourtellotte, W.G., Zhang, Y., AlemanMuench, G.R., Lewis, G., Chen, W., et al. (2021). Metformin inhibition of mitochondrial ATP and DNA synthesis abrogates NLRP3 inflammasome activation and pulmonary inflammation. Immunity 54, 14631477.e1411. 
51. Yang, L., Xie, X., Tu, Z., Fu, J., Xu, D., and Zhou, Y. (2021). The signal pathways and treatment of cytokine storm in COVID-19. Signal Transduct. Target. Ther. 6, 255.

52. Yue, Y., Nabar, N.R., Shi, C.S., Kamenyeva, O., Xiao, X., Hwang, I.Y., Wang, M., and Kehrl, J.H. (2018). SARS-Coronavirus open reading frame-3a drives multimodal necrotic cell death. Cell Death Dis. 9, 904.

53. Zhang, M., He, Q., Chen, G., and Li, P.A. (2020). Suppression of NLRP3 inflammasome, pyroptosis, and cell death by NIM811 in rotenone-exposed cells as an in vitro model of Parkinson's disease. Neuro-degenerative diseases 20,73-83.

54. Zhang, X., Liu, Y., Liu, J., Bailey, A.L., Plante, K.S., Plante, J.A., Zou, J., Xia, H., Bopp, N.E., Aguilar, P.V., et al. (2021). A trans-complementation system for SARS-CoV-2 recapitulates authentic viral replication without virulence. Cell 84, 2229-2238.e2213.

55. Zhong, Z., Liang, S., Sanchez-Lopez, E., He, F., Shalapour, S., Lin, X.J., Wong, J., Ding, S., Seki, E., Schnabl, B., et al. (2018). New mitochondrial DNA synthesis enables NLRP3 inflammasome activation. Nature 560, 198-203. 


\section{SARS-COV-2 VIROPORINS ACTIVATE THE NLRP3-INFLAMMASOME VIA THE MITOCHONDRIAL PERMEABILITY TRANSITION PORE}

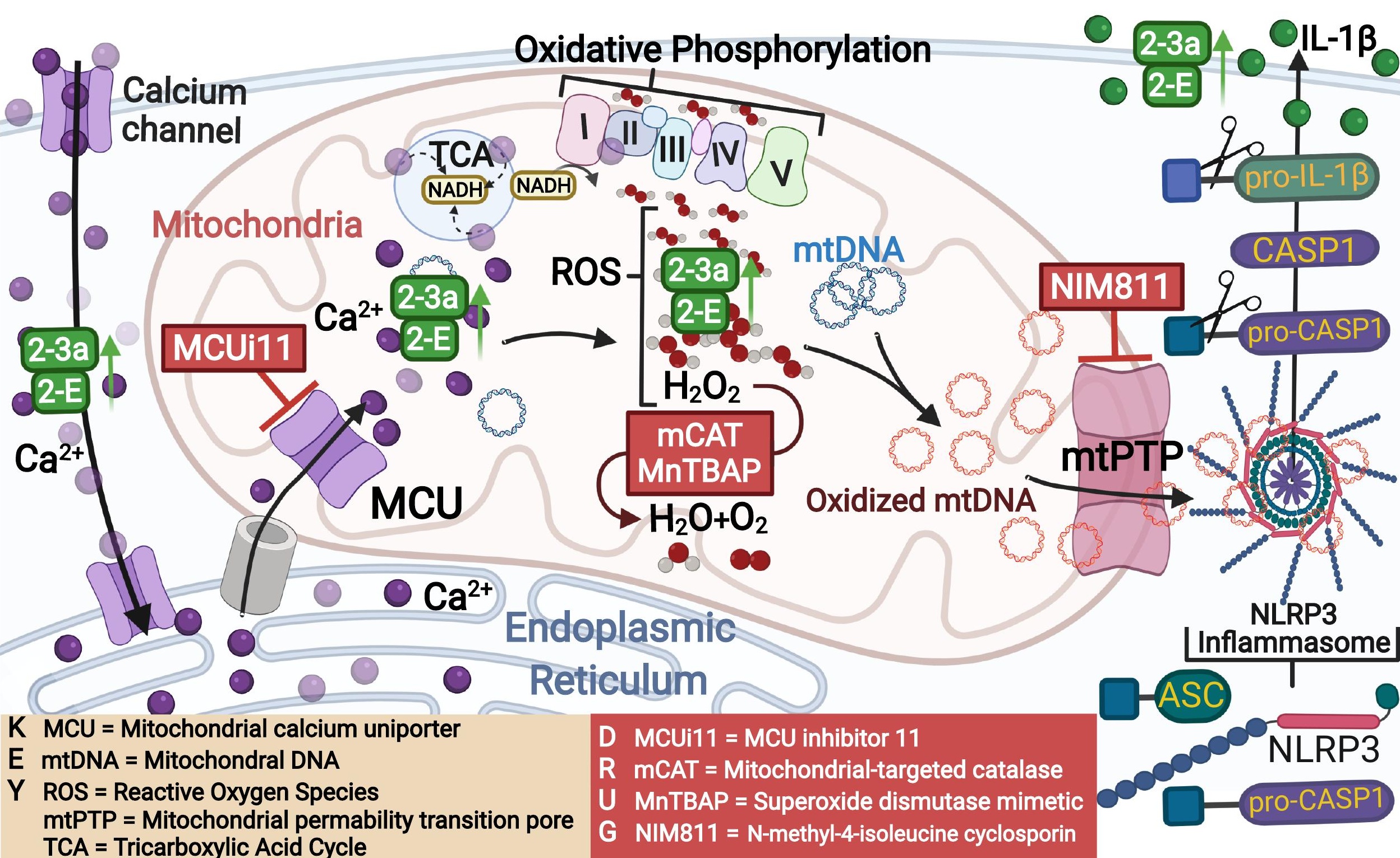



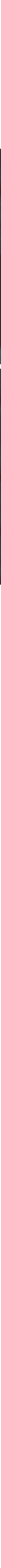

Figure 1. Expression of 2-E+2-3a induces mROS production by elevating mitochondrial $\mathrm{Ca}^{++}$ levels. 


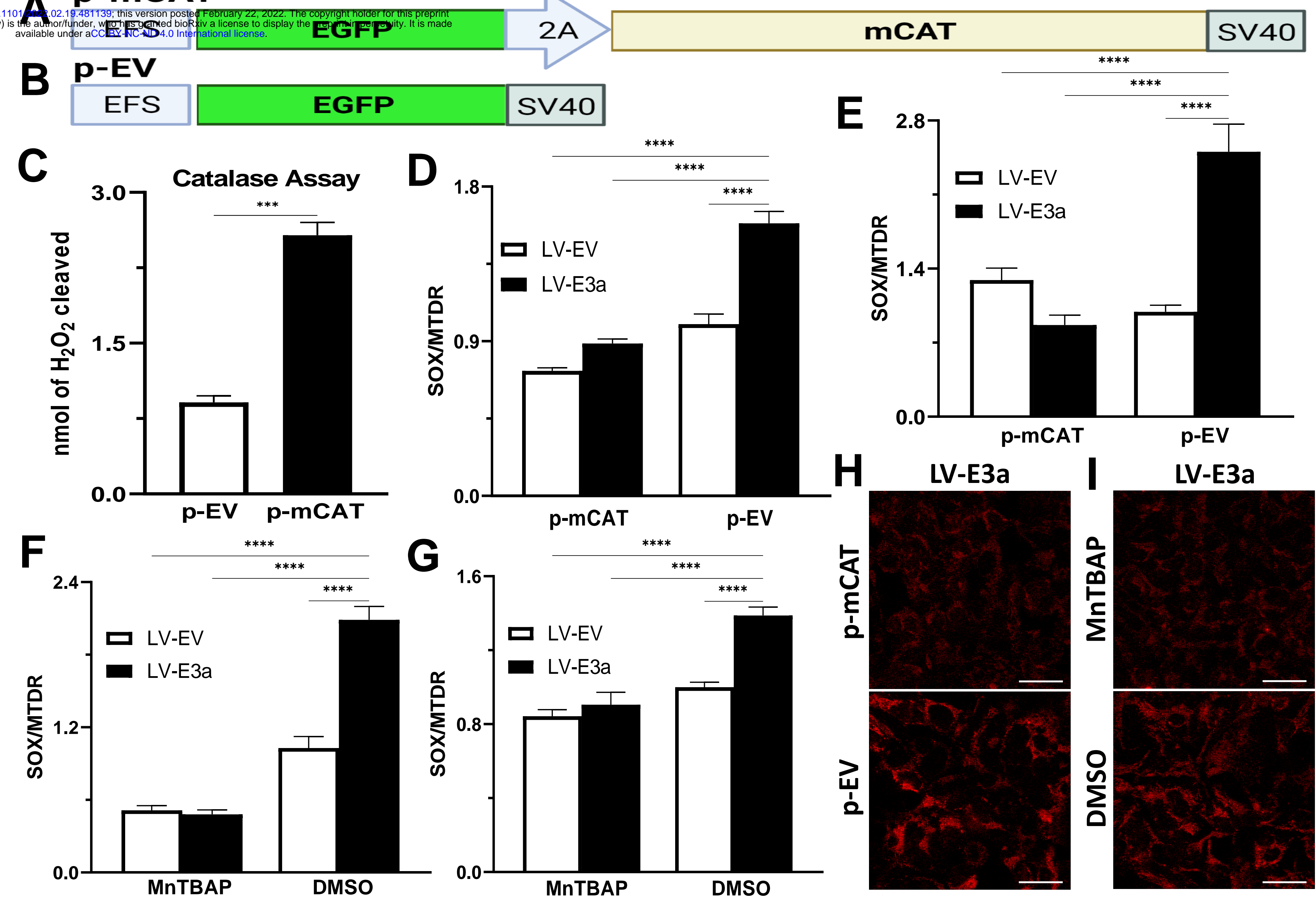

Figure 2. Expression of mCAT or treatment with the mROS scavenger MnTBAP antioxidant defenses blocks 2-E+2-3a induced $\mathrm{mROS}$. 


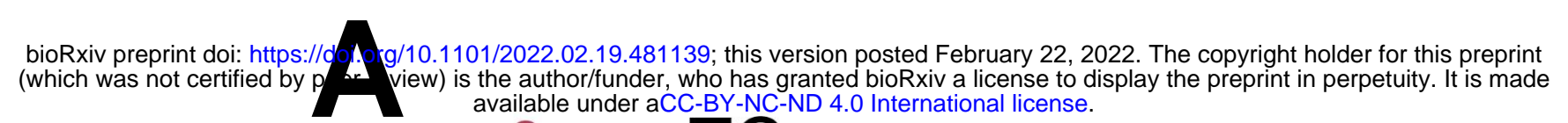
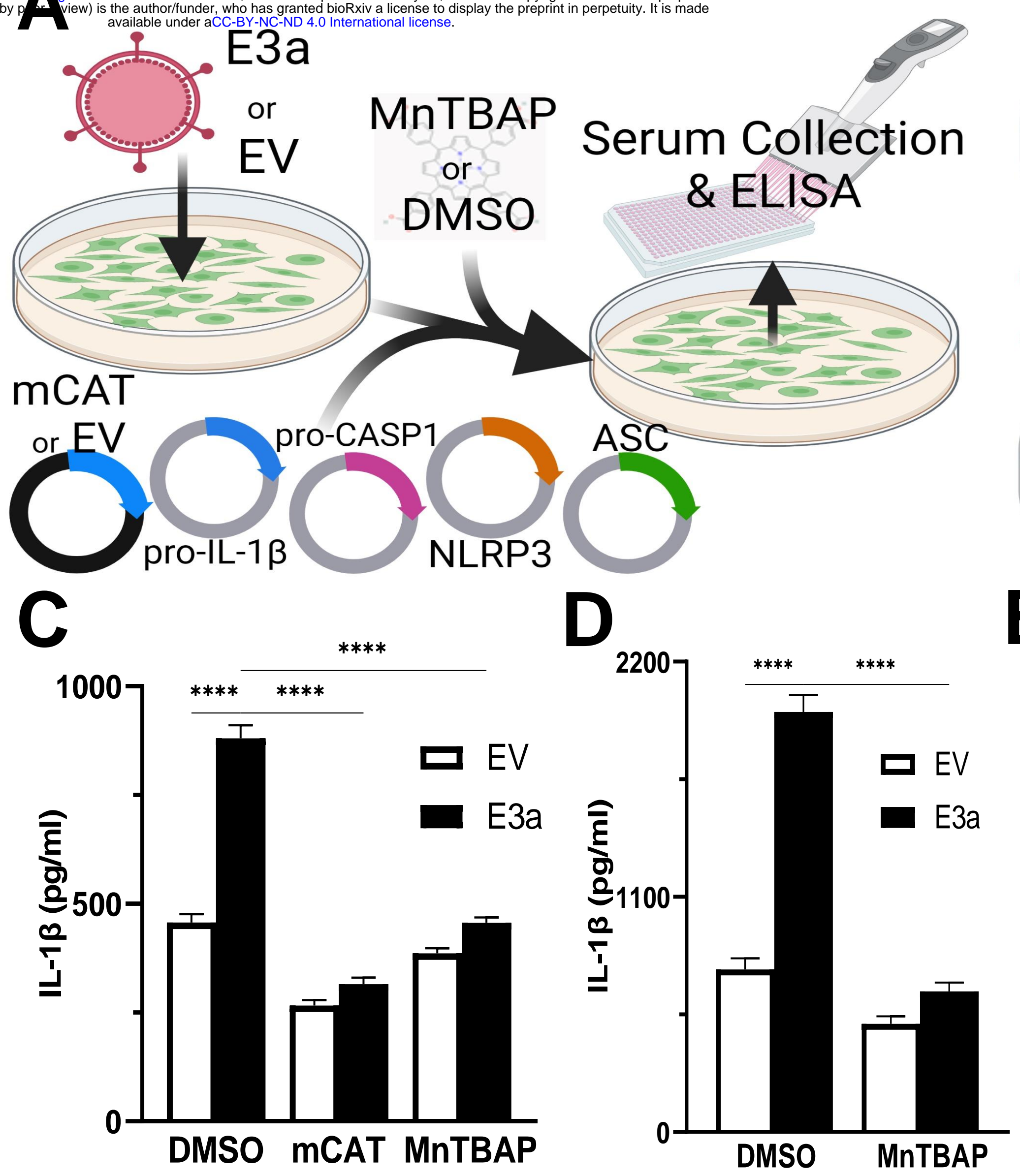

\section{THP1 Monocyte}

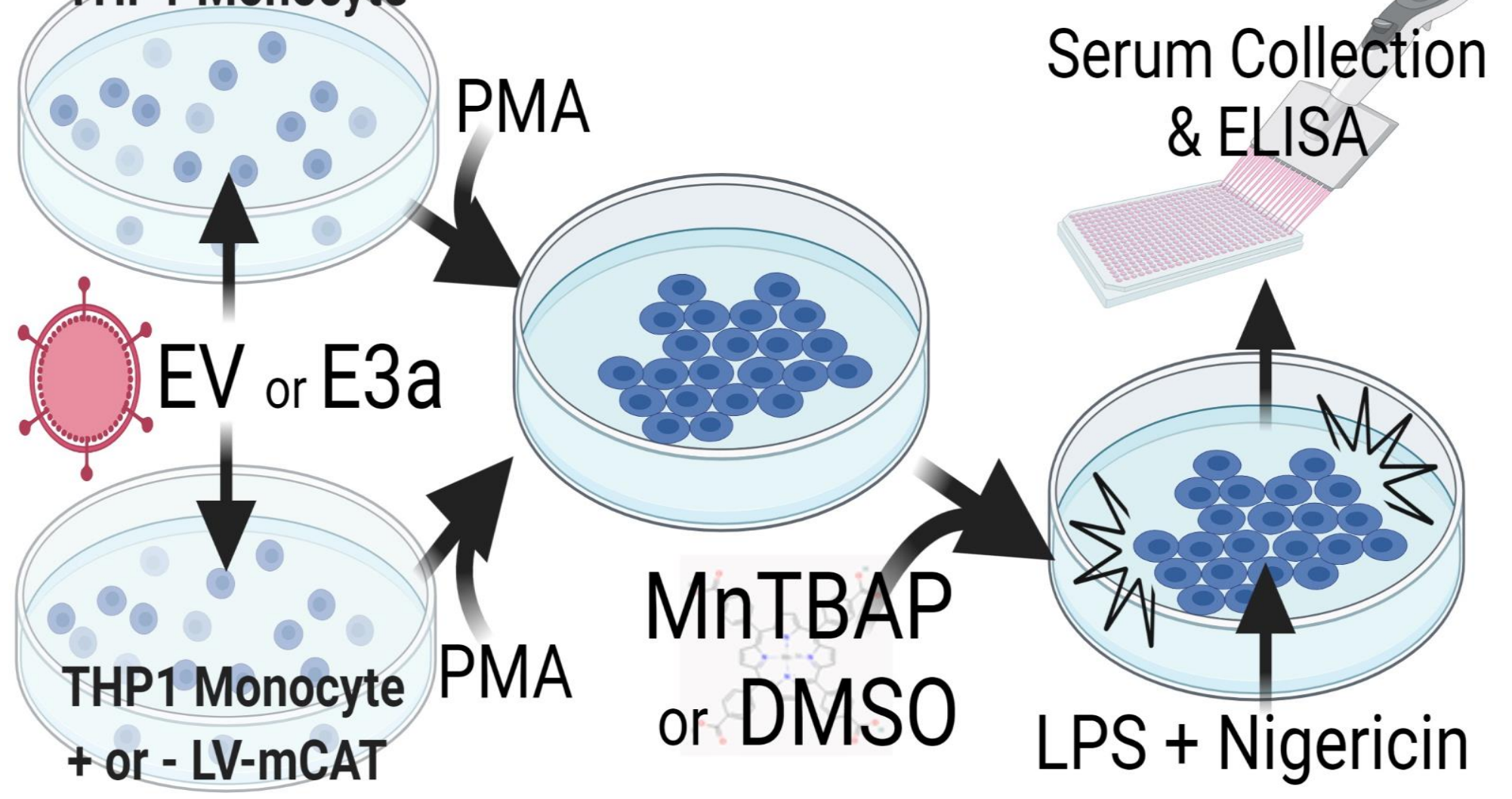

$\mathbf{E}$

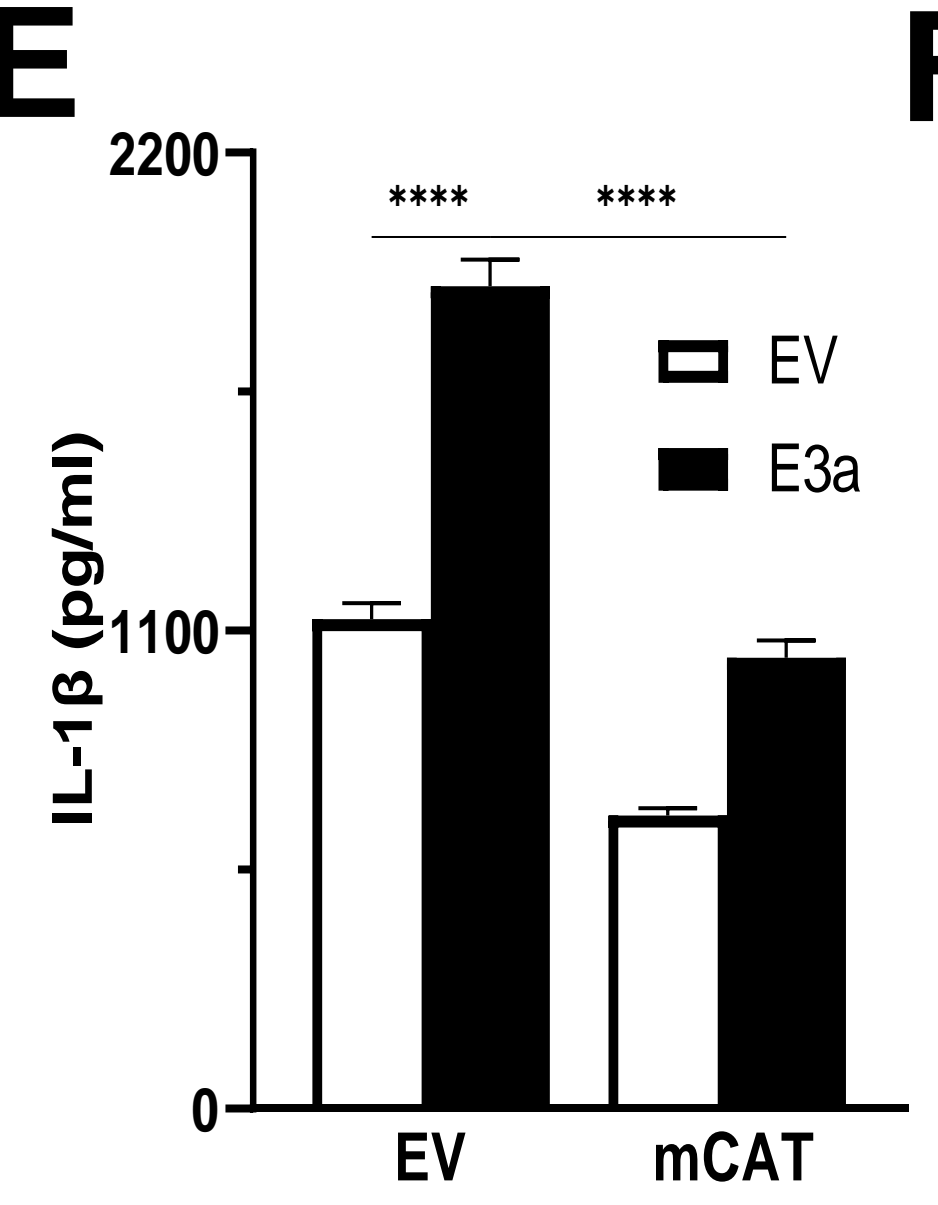

$\mathbf{F}$

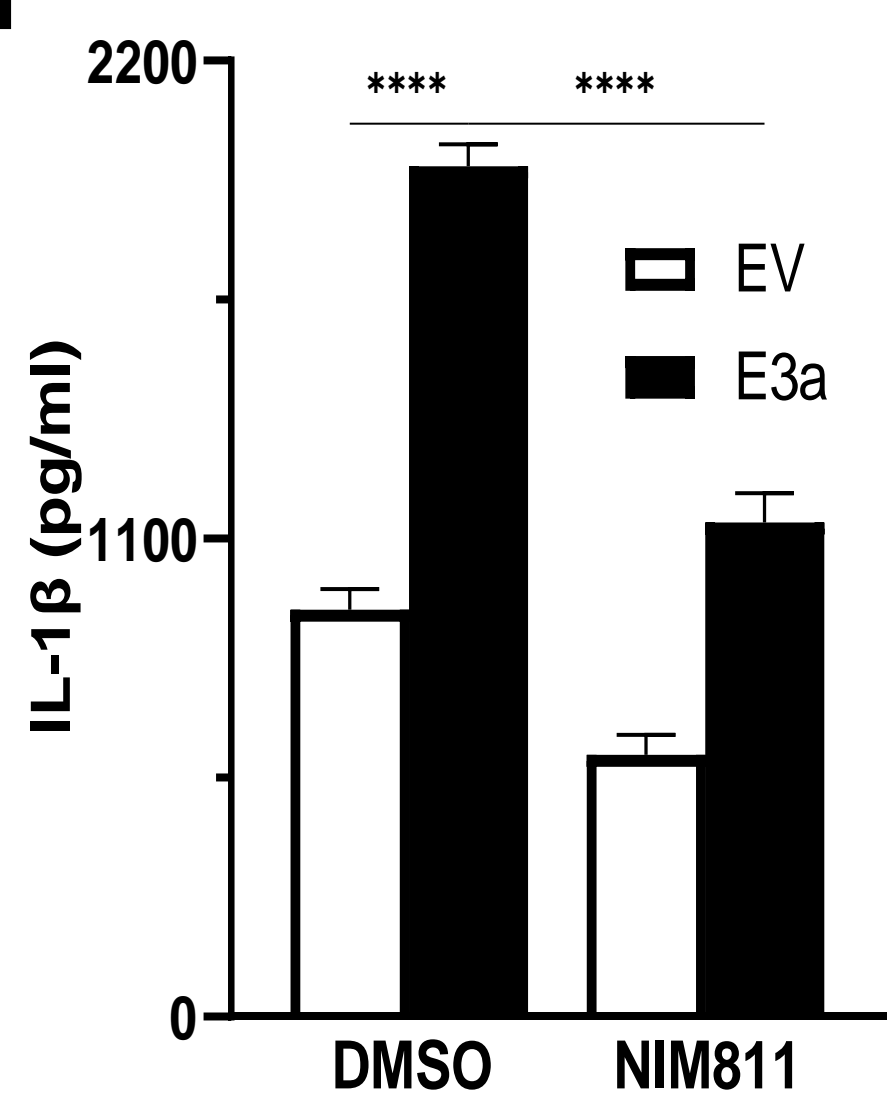

Figure 3. mROS and the mtPTP are required for activation of the NLRP3-I by the 2-E+2-3a viroporins. 